\title{
Theoretical and Experimental Exploration of Organic Synthesis Routes to Obtain Natural Rubber Analogues
}

\author{
David Mauricio Ramirez Sánchez ${ }^{1 * \dagger}$, Danilo González Forero ${ }^{2 \dagger}$, Pablo
} Ramos $^{3}$, Luis García Sánchez ${ }^{4}$

${ }^{1}$ Centro de Bioinformatica y Simulación Molecular, Universidad de Talca, Talca, Chile. *davramirez@utalca.cl

${ }^{2}$ Departamento de Química, Facultad de Ciencias, Universidad Nacional de Colombia, Bogotá, Colombia.dgnzlzf@gmail.com

${ }^{3}$ Theoretical Chemistry, Rutgers University, Newark, United States of America. prr34@pegasus.rutgers.edu

${ }^{4}$ Proyecto Curricular Licenciatura en Química, Facultad de Ciencias y Educación, Universidad

Distrital Francisco Jose de Caldas, Bogotá, Colombia. luisgracia@ctb.edu.co

${ }^{\dagger}$ These authors contributed equaly to this work

\section{Abstrac}

In the present work synthesis routes and the reactions involved in the polymerization of two organic compounds similar to natural rubber were studied. To these routes and reactions, modifications related with the previously reported in the literature were performed in order to study them through theoretical tools and determine their prediction capability for the design of those analogs. The semiempirical method Austin Model 1 (AM1) was used for the study of electronic configurations and it was shown that the synthesis of the polystyrene polymer (PS) and the poly copolymer (styrene-butadiene) (SBR) is possible through the use of free radicals from styrene and 1,3-butadiene monomers using benzoyl peroxide as the catalyst in both cases; going through the intermediates 1-phenyl-2-[(phenylcarbonyl)oxy]ethyl and (2E)-4(oxy (phenylcarbonyl))2buten-1-yl. The results show that the higher electronic densities are found over the active atom in the radical compared to the electronic densities over the ions and the interest atoms in a possible condensation. Additionally, the study of the reaction mechanisms for the designed organic synthesis routes was performed 
by applying the frontier orbitals theory developed by R. B. Woodward, and R. Hoffmann and the respective correlation diagrams that showed thermal viability were built demonstrating the consistency between the characteristics of the orbital symmetry in the concerted reactions of the reactants and products; therefore it is seen that the free radical synthesis do not show symmetry restrictions, and it is possible to make it by a thermal route with low activation energy thresholds. The experimental yields of polymerizations were $90 \%$ and 75\% for polystyrene (PS) and poly (styrene-butadiene) (SBR) respectively. Additionally mechanical tests were performed to the synthesized polymers and it was proved that the properties of the synthesized compounds are consistent with those reported in the literature. In the future it is expected to explore unknown organic synthetic routes with this research it was demonstrated that the methods are reliable.

\section{Keywords}

Semiempirical method AM1, Frontier orbitals theory, Polymerization theoretical study, Synthesis of SBR and PS.

\section{Introduction}

Polymerization reactions can be classified in five different categories as follows: Condensation polymerization, this one consists in the elimination of a water molecule between two reactants to create a new bond ${ }^{6}$; the ionic polymerization divided in catatonic polymerization and anionic polymerization where they are catalyzed by cations and anions respectively ${ }^{5}$; the polymerization by free radicals is the creation of an initial free radical that allows at the same time the creation of another free radical when reacting with the compound ${ }^{5}$. This kind of polymerization was the selected one to develop the synthesis routes. The photo polymerization makes reference to the initiated by light polymerization generally used in dental resin industry ${ }^{15}$; radio polymerization is based in the polymerization mechanism that happens when using gamma radiation as free radicals former agent ${ }^{14}$.

Different means to carry out polymerizations are: Emulsion; this consist in adding emulsifier agents and other additives to obtain the polymer as a stable dispersion in aqueous mean with aqueous properties. In this mean, polymerization was the selected one for this study because it fulfills the general characteristics of natural latex ${ }^{10}$. Polymerization in solution consists in 
obtain low viscosity solutions, allowing the easy heat dissipation and avoiding the overheating in specific zones. Besides, it allows a good control of polymer molecular weight and prevents the interweaving ${ }^{1}$. In suspension polymerization, the monomers or the monomer mix is swarmed by a drop shaped strong agitation in a second liquid phase; where both, monomers and polymer, are unsolvable ${ }^{13}$.

In 1985, Dewar and collaborators designed a new algorithm called "Austin Model1". This has been parameterized for the first and second row elements including the $\mathrm{Zn}, \mathrm{Hg}$ and $\mathrm{H}^{4}$. This method adds new exponents to each atom in a molecule and adds a repulsion function given by:

$f_{A B}^{A M 1}=f_{A B}^{M N D O}+\frac{C_{A} C_{B}}{R_{A B} / A}\left[\sum_{k} a_{k A} \exp \left[-b_{k A}\left(R_{A B}-c_{k A}\right)^{2}\right]+\sum_{k} a_{k B} \exp \left[-b_{k B}\left(R_{A B}-c_{k B}\right)^{2}\right]\right]$

Where:

$$
f_{A B}^{M N D O}=C_{A} C_{B}\left(S_{A} S_{A} \mid S_{B} S_{B}\right)\left(e^{-\alpha_{A} R_{A B}}+e^{-\alpha_{B} R_{A B}}\right)
$$

$f_{A B}^{M N D O}$ is the core-core repulsion term, $C_{A}$ y $C_{B}$ are the core charges of the atoms $A$ and $B$, $\alpha_{A}, \alpha_{B}, a_{k A}, b_{k A}$ and $c_{k A}$ are the parameters.

With this core function new parameterizations were acquired related to the ones acquired by old methods. With this method the approximation is greater allowing a better manipulation in its algorithm.

The study of reaction mechanisms to organic chemistry was broadly studied by R. B. Woodward and R. Hoffmann ${ }^{7}$ in the middle 60's stating the following basic principle: Reactions happen easily when there is cogency between the orbital symmetry characteristics of reagents and products, and difficulty when this cogency does not exist. Properly said: in arranged reactions orbital symmetry is preserved ${ }^{11}$. Concerted reactions are those that happen in one step, it means, all the bonds are broken and formed at the same time; so that the electron reordering occur in a continuous step.

\section{Methods}

The research was approached from two central points: the theoretical study of the possible polimer synthesis route or routes with similar properties to natural rubber ${ }^{17}$, through computational chemistry and the experimental testing of those results. Possible synthesis were simulated with semi-empirical method AM1 previous pencil and paper analysis applying the groups theory. 


\section{1,3-butadiene monomer synthesis}

1,3-butadiene monomer synthesis was realized starting from succinic acid ${ }^{9}$, which was conjugated with ethanol in sulfuric acid medium acquiring etil succinate. This was reduced by Bolveaut-Blanc method ${ }^{18}$ to obtain 1,4-butanediol, which was dehydrate with sulfuric acid to produce 1,3-butadiene, as shown in Figure 1.

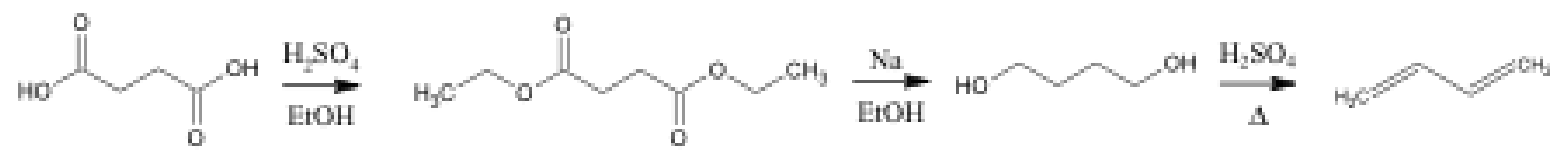

Figure 1: 1,3-butadiene synthesis

\section{PS (Polystyrene) synthesis}

Polystyrene synthesis was made by free radicals ${ }^{3}$, using benzoyl peroxide as initiator, which by thermical decomposition generate the phenylcarbonyl radical (Figure 2), which reacts with a styrene molecule generating the radical 1-phenyl-2 [(phenylcarbonyl)oxy]ethyl (Figure 3). Starting polymerization which ends when two radicals are found, as shown in Figure 4.

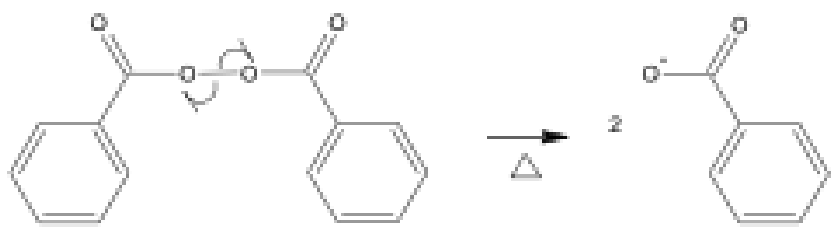

Figure 2: Thermical decompositon of benzoyl peroxide

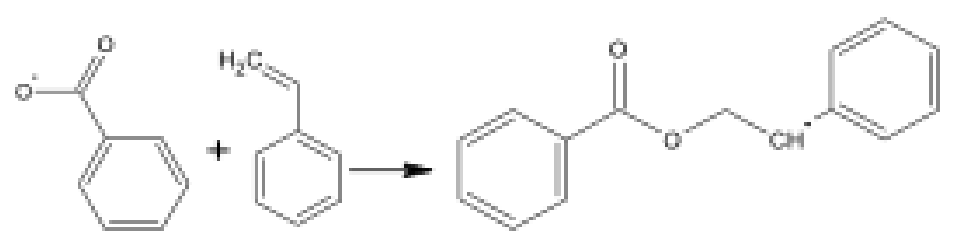

Figure 3: 1-phenyl-2-[(phenylcarbonyl)oxy]ethyl radical creation. Polymeric chain spreading 

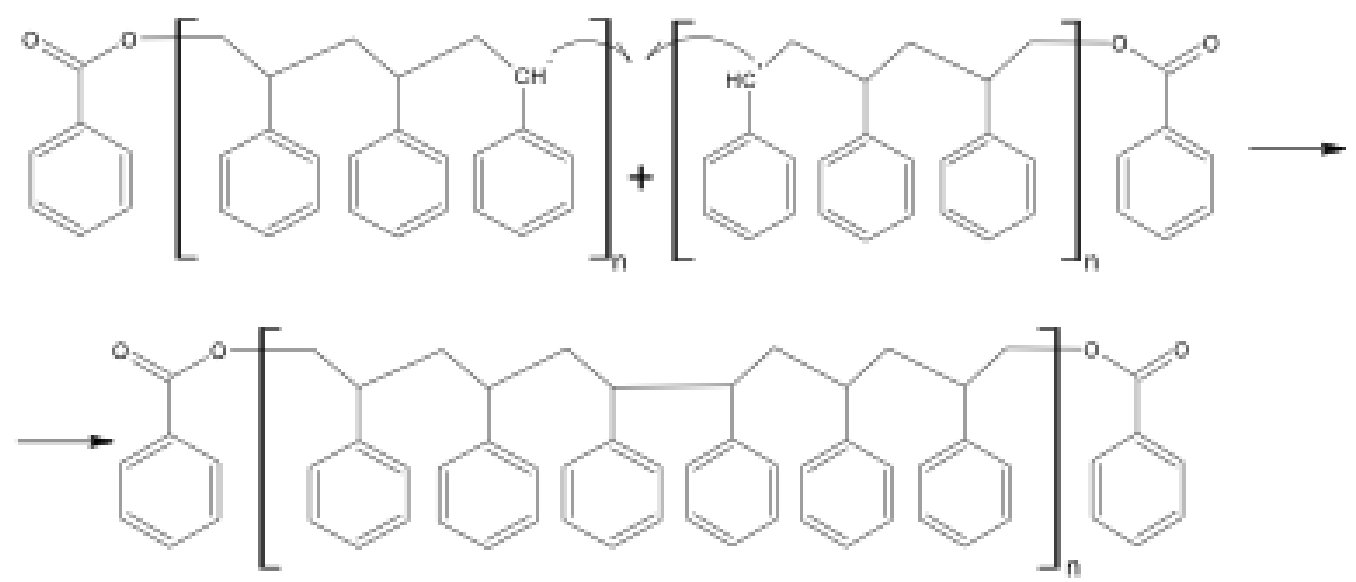

Figure 4: 1-phenyl-2-[(phenylcarbonyl)oxy]ethyl radical creation. Polymeric chain spreading

\section{SBR (Styrene-butadiene copolymer) synthesis}

Copolymer synthesis was made by using as monomers styrene and 1,3-butadiene previously synthetized. A styrene emulsion was prepared, adding 1.3-butadiene and benzoyl peroxide as starter ${ }^{17}$. Sodium palmitate was used as emulsifier. This mixture was treated with agitation and constant heating during 5 hours. Thereupon the ending and crosslinking agent (hydroquinone) was added.

\section{Results and Discussion}

The semiempirical method Austin Model 1 (AM1) was used for the study of electronic configurations and it was shown that the synthesis of the polystyrene polymer (PS) and the poly copolymer (styrene-butadiene) (SBR) is possible through the use of free radicals from styrene and 1,3-butadiene monomers using benzoyl peroxide as the catalyst in both cases; going through the intermediates 1-phenyl-2-[(phenylcarbonyl)oxy]ethyl and (2E)-4(oxy (phenylcarbonyl))2buten-1-yl.

The theoretical study, using semi-empirical method to synthesize of 1,3-butadiene was developed by an analysis from succinic acid, its transformation to ester, reduction to diol and alquenación reaction in acid medium; with an overall yield of $72 \%$. Being the succinic acid molecule symetric, wave functions were assigned to only one of the acids. (Figure 5).

Likewise, to the carbon and oxygen atoms that participate in the reaction from ethanol wave functions were assigned.

The semiempirical estimate argue the symmetry analysis developed, because the HOMO 


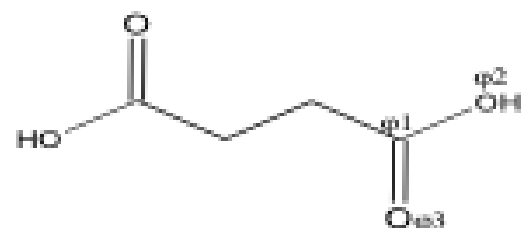

Figure 5: Wave functions selected for the succinic acid molecule

from ethanol shows that oxygen of its molecule contains the electronic charge ready to be transferred to an electrophile; in other words, the conclution by the acid's LUMO orbital indicates that the reaction is evidently given between the nucleophilic ethanol (oxygen) zone and the electrophilic (carbonyl carbon) acid part ${ }^{2}$. This interaction concludes in the acetate succinate whose configuration has a lower energy orbital and is allowed by symmetry.

The last given reaction in this stage of the monomer synthesis (1,3-butadiene), shows that the sequence of reactivity decreases and the stability sequence increases. However the monomer is the appropriate one because its orbital configuration is distributed in secular energies separated by the unit. This characteristic is favorable for polymerizing conjugated diene due to the ease way to increase the electron charge to higher energy levels, and hence more likely to the reaction. This tendency is observed in the analysis of the polymerization reaction where the diene raises its load due to the action of highly exothermic compounds in reaction.

The values of the LUMO orbital of benzoyl peroxide where the homolytic breaking is created due to thermal action are observed. Therefore these two atoms are the interesting ones in this reaction. These values indicate that the first occupied orbitals are willing to donate electrons, afterwards we can conclude that the break happens in a homolytic way as predicted (Figure 2).

The generated radical has more negative power settings; therefore it can not be said that it is more stable. According to quantum mechanics it is possible to obtain negative energies due to the amount of electronic energy located in the molecule, this is the reason why when more negative the oxidanilo radical energies are, more favorable is to give all his electronic charge and seek a more stable electronic configuration.

In styrene analysis by symmetry, carbons that are not part of the aromatic ring were taken. Although this influences the spatial distribution of the product, does not have significant influence on the high speed at which the polymerization reaction occurs. Orbital analysis indicates and argues the fact that the radical will give all its charge to styrene primary carbon because the selected $\pi$ electrons are highly reactive and willing to interact with the radical's free electron facilitating sigma bond, and the unpaired electron forms the new radical.

The formation of the 1-phenyl-2-[(phenylcarbonyl) oxy] acetate radical is shown in Figure 3; 
where the oxidanilo radical (phenylcarbonyl) attacks the primary carbon of the styrene molecule, initiating polymerization. Reaction is predicted by this carbon, according to the computational study conducted since it indicates that this carbon is the most willing one to receive electrons. Due to that, only the directly involved atoms are taken in the reaction, the modified molecule to be analyzed belongs to the symmetry point group $C_{s}$. This molecule is shown in Figure 6, which indicates which wave functions were selected.

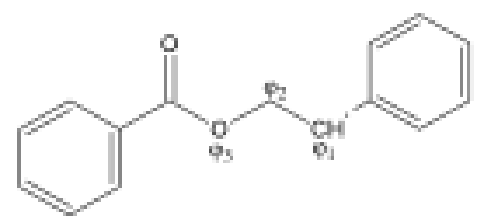

Figure 6: Wave functiones selected to 1-phenyl-2-[(pheniycarboniy)oxy]ethyl radical

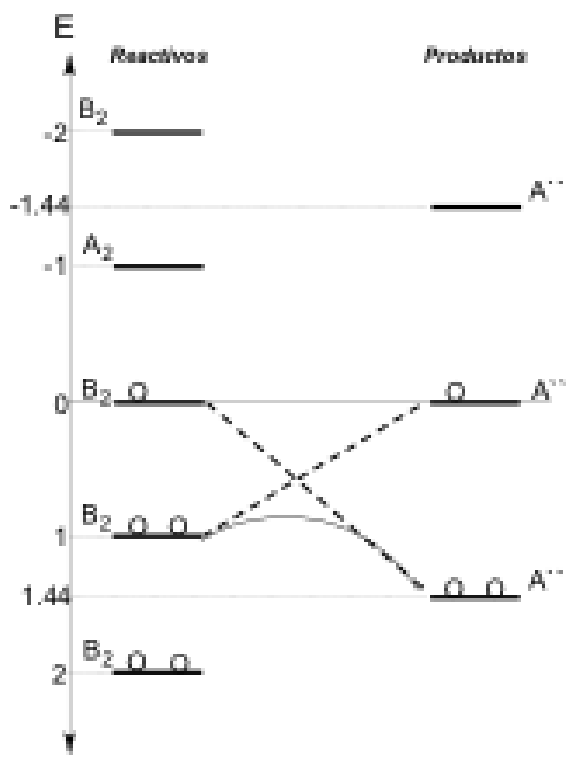

Figure 7: Correlation diagram to form 1-phenyl-2-[(pheniycarboniy)oxy]ethyl radical

As seen in Figure 7, the reaction between the phenylcarbonyl radical and styrene is thermally allowed for obtaining radical 1-phenyl-2-[(phenylcarbonyl)oxy]ethyl; because in the decay of electrons found in orbital $B_{2}$ to $A$ " photochemical excitation is not required because it is not necessary to overcome the energetic barrier (represented by the value "zero"). Moreover, note that the energies corresponding to the carbonyl group of the oxidanilo radical (phenylcarbonyl) 
do not have relevance in the reaction, since the electrons do not change or interfere with this one at any time, because the carbonyl group is preserved; but these $\pi$ electrons do influence in the radical stability, because they generate a resonant effect among them and the other $\pi$ electron.

SBR copolymerization was studied in the same way that the styrene polymerization ${ }^{16}$. The reaction is shown in Figure 8. The decomposition of the benzoyl peroxide to generate phenylcarbonyl radical is shown in Figure 2, and fullfils the same principle as in the styrene polymerization. This radical will mainly attack the primary carbons of 1,3-butadiene because these carbons are more willing to receive electrons (Table 1), generating the (2E) -4 - [oxy (phenylcarbonyl)]-2-buten-1 -yl radical, which reacts with a molecule of the styrene generating the (2E)-6-phenyl-1-[oxy (phenylcarbonyl)]-2-hexen-6-yl radical giving way to the propagation of the polymerization step.

\begin{tabular}{|c|c|c|c|c|c|}
\hline \multirow[t]{2}{*}{ Molecules } & \multirow{2}{*}{$\begin{array}{l}\text { Symmetry } \\
\text { Group }\end{array}$} & \multirow{2}{*}{$\begin{array}{l}\text { Secular Energy } \\
\qquad(\beta)\end{array}$} & \multirow{2}{*}{$\begin{array}{l}\text { Total Energy } \\
\text { (Kcal/mol) }\end{array}$} & \multicolumn{2}{|c|}{ Orbital Energy $(\mathrm{eV})$} \\
\hline & & & & HOMO & LUMO \\
\hline Styrene & $C_{2 v}$ & $E_{1}=1 ; E_{2}=-1$ & -1745.4256 & -8.9977 & 0.0196 \\
\hline 1,3-butadiene & $C_{2 v}$ & $\begin{array}{c}E_{1}=0.62 ; E_{2}=1.62 ; \\
E_{3}=-0.62 ; E_{4}=-1.62\end{array}$ & -966.3816 & -9.3343 & 0.4491 \\
\hline PS & $C_{s}$ & $E_{1}=1 ; E_{2}=-1$ & \multicolumn{3}{|c|}{ Non calculated } \\
\hline SBR & $C_{1}$ & $E_{1}=1 ; E_{2}=-1$ & \multicolumn{3}{|c|}{ Non calculated } \\
\hline 1,4-butanediol & $C_{s}$ & $E_{1}=1 ; E_{2}=-1$ & -.1445 .3850 & -10.9150 & 3.1409 \\
\hline Ethyl Succinate & $C_{s}$ & $\begin{array}{c}E_{1}=0 ; E_{2}=1.44 \\
E_{3}=-1.44\end{array}$ & -2532.8323 & -11.3289 & 0.9572 \\
\hline Succinct acid & $C_{s}$ & $E_{1}=1.44 ; E_{2}=-1.44$ & -4185.4916 & -11.4721 & 0.6858 \\
\hline Ethanol & $C_{s}$ & $E_{1}=1 ; E_{2}=-1$ & -776.7216 & -10.8772 & 3.5645 \\
\hline Water & $C_{2 v}$ & $\begin{array}{c}E_{1}=0 ; E_{2}=2 \\
E_{3}=-2\end{array}$ & -223.0294 & -12.4644 & 4.4187 \\
\hline Benzoyl Peroxide & $C_{2 v}$ & $E_{1}=1 ; E_{2}=-1$ & -3176.5634 & -10.2057 & -0.7235 \\
\hline Phenylcarbnyl radical & $C_{2 v}$ & $\begin{array}{c}E_{1}=0 ; E_{2}=2 \\
E_{3}=-2\end{array}$ & -1570.6697 & -10.3386 & -8.0466 \\
\hline $\begin{array}{l}\text { 1-phenyl-2-[(phenyl- } \\
\text { carboniyl)oxy]ethyl radical }\end{array}$ & $C_{s}$ & $\begin{array}{c}E_{1}=0 ; E_{2}=1.44 \\
E_{3}=-1.44\end{array}$ & -3364.7824 & -9.8480 & -4.4832 \\
\hline $\begin{array}{l}\text { (2E)-4-[oxy(phenyl-carbonyl)] } \\
\text {-2-buten-1-yl radical }\end{array}$ & $C_{s}$ & $\begin{array}{c}E_{1}=0 ; E_{2}=1.44 \\
E_{3}=-1.44\end{array}$ & -2592.6426 & -10.0387 & -4.3846 \\
\hline $\begin{array}{l}\text { (2E)-6-phenyl-1-[oxy(phenyl } \\
\text {-carbonyl)]-2-hexen-6-yl radical }\end{array}$ & $C_{s}$ & $\begin{array}{c}E_{1}=0 ; E_{2}=1.44 \\
E_{3}=-1.44\end{array}$ & -4643.7710 & -9.7775 & -4.2894 \\
\hline Hydroquinone & $C_{2 v}$ & $\begin{array}{l}E_{1}=1 ; E_{2}=-1 \\
E_{3}=1 ; E_{4}=-1\end{array}$ & -1522.8928 & -8.7334 & 0.2343 \\
\hline
\end{tabular}

Table 1: Results of theoretical studies

Completion and crosslinking was performed using hydroquinone (Figure 9), where a growing polymer chain reacts with the hydroquinone generating a radical 1 , which can generate another radical by electronic rearrangement. These radicals can react with an unsaturation of a 

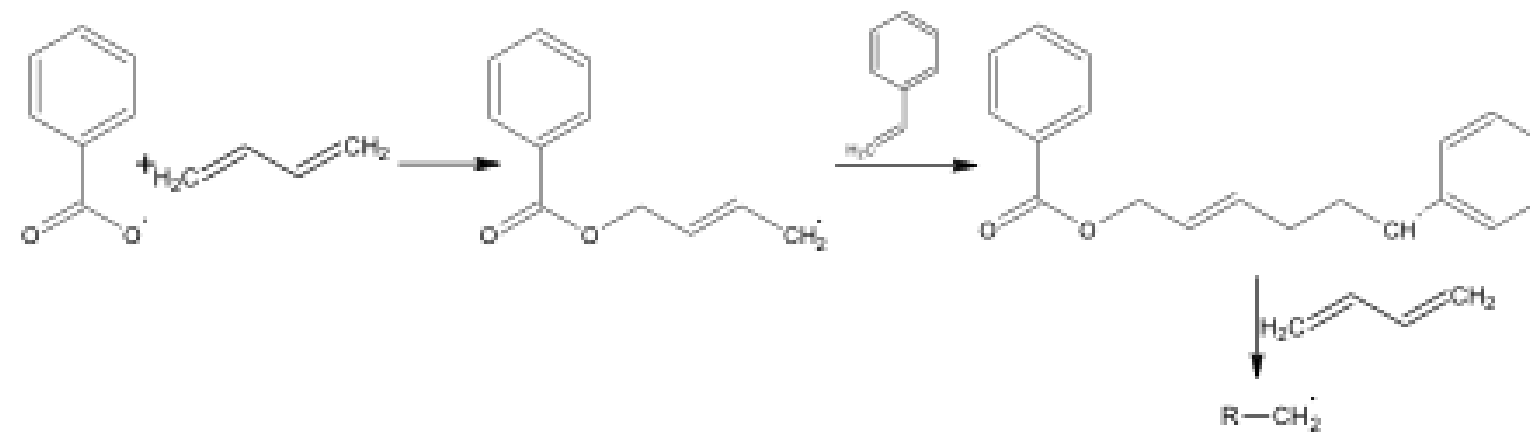

Figure 8: Iniciation and propagation to generate the copolimeric unsaturated chain growing

terminated string by the reaction of two growing chains generating a radical 2 where you can see how is the crosslinking of the copolymer taking place. This radical 2 reacts with another growing chain to generate the SBR, or it can react with another string finalized. The reactions are given when there are completed and growing polymeric chains, until the crosslinking cup reaches its maximum.

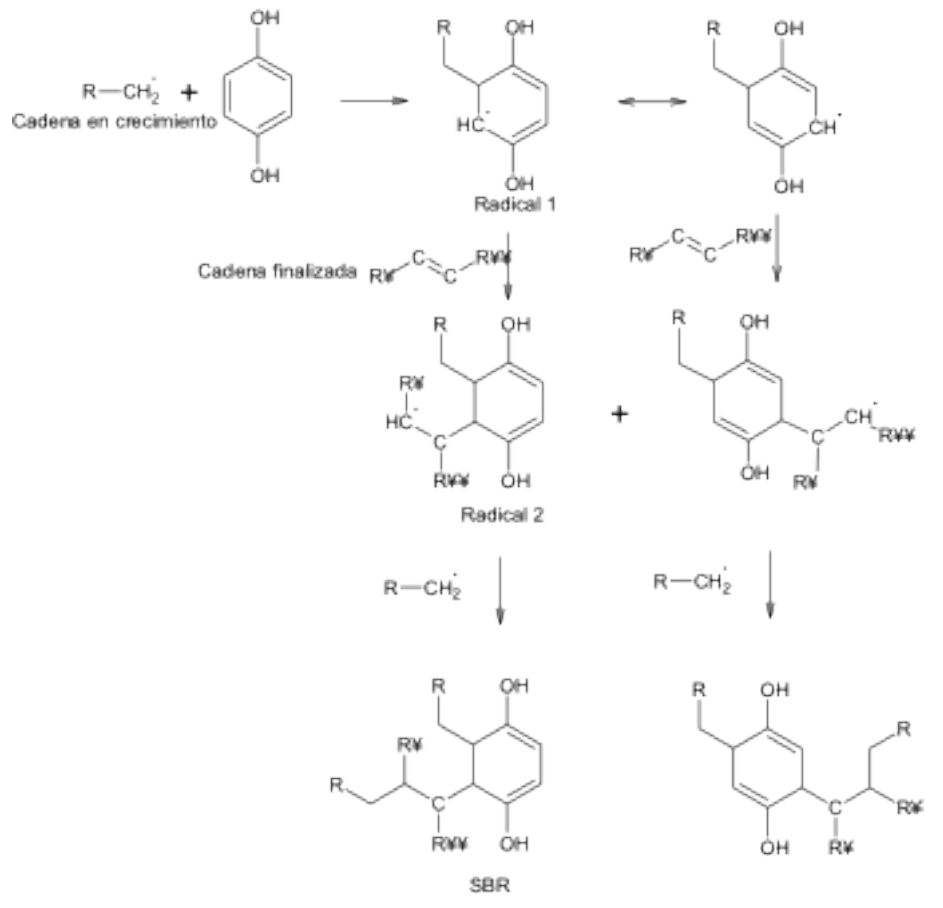

Figure 9: Crosslinking and SBR finalization

Polymerizations yields were, in the case of polystyrene, about $90 \%$; in the case of the copolymer about $70 \%$. The synthesized polymers properties correspond to those reported in the lit- 
erature $^{12}$. In the case of polystyrene its rigidity, hardness and transparency were highligthed, in the case of the styrene-butadiene copolymer was its elasticity. It should be noted that these properties were not quantified, because the sums were not sufficient for developing technical tests established by the ASTM guidelines ${ }^{8}$.

\section{Conclusions}

Two theoretical studies of a polymerization reaction were carried out for obtaining an analog to natural rubber. The semi-empirical AM1 method indicates that it is possible to synthesize polystyrene and poly (styrene-butadiene) from styrene and 1,3-butadiene using benzoyl peroxide as the catalyst in both cases, through the intermediates 1-phenyl-2-[(phenylcarbonyl)oxy]ethyl and (2E)-4(oxy (phenylcarbonyl)) 2-buten-1-y. The greater electronic density is presented on the radical oxygen atom from phenylcarbonyl radical; the initiator of the reaction. In 1,3-butadiene as well as in styrene, the most willing carbons to receive electrons are the primary carbons in the molecule; these carbons act as electrophiles receiving the oxidanilo's free electron (phenylcarbonyl).

The application of groups theory shows that the reactions between the above species are allowed by the symmetry of the electronic states involved favoring the product formation via thermal with a low threshold activation energy.

The theoretical results were experimentally verified, obtained yields of $90 \%$ for polystyrene and $70 \%$ for styrene-butadiene copolymer.

\section{Acknowledgements}

The authors would like to express their gratitude to Josue García for the valuable contribution to this work. To the Universidad Francisco josé de Caldas District for providing adequate space for this research.

\section{Referencias}

1. L. S. Boffa and B. M. Novak. Copolymerization of polar monomers with olefins using transition-metal complexes. Chemical reviews, 100(4):1479-1494, 2000. 
2. M. J. Castaldi and E. Kwon. An investigation into the mechanisms for styrene-butadiene copolymer (sbr) conversion in combustion and gasification environments. International journal of green energy, 4(1):45-63, 2007.

3. E. Cloutet, J.-L. Fillaut, D. Astruc, and Y. Gnanou. Newly designed star-shaped polystyrene: Synthesis and characterization. Macromolecules, 31(20):6748-6755, 1998.

4. M. J. Dewar, E. G. Zoebisch, E. F. Healy, and J. J. Stewart. Development and use of quantum mechanical molecular models. 76. am1: a new general purpose quantum mechanical molecular model. Journal of the American Chemical Society, 107(13):3902-3909, 1985.

5. A. Goodall, M. Wilkinson, and J. Hearn. Mechanism of emulsion polymerization of styrene in soap-free systems. Journal of polymer science: Polymer chemistry edition, 15(9):2193$2218,1977$.

6. J. Hine. Physical organic chemistry, volume 43. McGraw-Hill New York, 1962.

7. R. Hoffmann and R. B. Woodward. Conservation of orbital symmetry. Accounts of Chemical Research, 1(1):17-22, 1968.

8. Q. Q. Ke, X. Y. Huang, P. Wei, G. L. Wang, and P. K. Jiang. Thermal, mechanical, and dielectric behaviors of crosslinked linear low density polyethylene/polyolefin elastomers blends. Journal of applied polymer science, 104(3):1920-1927, 2007.

9. I. Kuntz. The copolymerization of 1,3-butadiene with styrene by butyllithium initiation. Journal of Polymer Science, 54(160):569-586, 1961.

10. E. W. Madge. Latex foam rubber. Maclaren, 1962.

11. J. McMurry. Quimica organica/Organic Chemistry. Cengage Learning Editores, 2008.

12. D. Panse and P. Phillips. Polymer data handbook, 1999.

13. F. Romani, E. Passaglia, M. Aglietto, and G. Ruggeri. Functionalization of sbr copolymer by free radical addition of thiols. Macromolecular Chemistry and Physics, 200(3):524-530, 1999.

14. D. A. Smith. Addition polymers: formation and characterization. Butterworth \& Co Publishers Ltd, 1968. 
15. V. Vlack. Elements Of Material Science And Engineering, 6/E. Pearson Education India, 1959.

16. A. I. Vogel. A textbook of practical organic chemistry including qualitative organic analysis. 1974.

17. B. Vollmert and E. Immergut. Polymer chemistry. Springer-Verlag New York, 1973.

18. G. S. Whitby. Synthetic rubber. J. Wiley, 1954. 